\title{
Inverse Spectral Problems for Tridiagonal $N$ by $N$ Complex Hamiltonians ${ }^{\star}$
}

Gusein Sh. GUSEINOV

Department of Mathematics, Atilim University, 06836 Incek, Ankara, Turkey

E-mail: guseinov@atilim.edu.tr

URL: http://www.atilim.edu.tr/ guseinov/

Received November 18, 2008, in final form February 09, 2009; Published online February 14, 2009 doi:10.3842/SIGMA.2009.018

\begin{abstract}
In this paper, the concept of generalized spectral function is introduced for finiteorder tridiagonal symmetric matrices (Jacobi matrices) with complex entries. The structure of the generalized spectral function is described in terms of spectral data consisting of the eigenvalues and normalizing numbers of the matrix. The inverse problems from generalized spectral function as well as from spectral data are investigated. In this way, a procedure for construction of complex tridiagonal matrices having real eigenvalues is obtained.
\end{abstract}

Key words: Jacobi matrix; difference equation; generalized spectral function; spectral data 2000 Mathematics Subject Classification: 15A29; 39A10

\section{Introduction}

Consider the $N \times N$ tridiagonal symmetric matrix (Jacobi matrix) with complex entries

$$
J=\left[\begin{array}{ccccccc}
b_{0} & a_{0} & 0 & \cdots & 0 & 0 & 0 \\
a_{0} & b_{1} & a_{1} & \cdots & 0 & 0 & 0 \\
0 & a_{1} & b_{2} & \cdots & 0 & 0 & 0 \\
\vdots & \vdots & \vdots & \ddots & \vdots & \vdots & \vdots \\
0 & 0 & 0 & \cdots & b_{N-3} & a_{N-3} & 0 \\
0 & 0 & 0 & \cdots & a_{N-3} & b_{N-2} & a_{N-2} \\
0 & 0 & 0 & \cdots & 0 & a_{N-2} & b_{N-1}
\end{array}\right]
$$

where for each $n, a_{n}$ and $b_{n}$ are arbitrary complex numbers such that $a_{n}$ is different from zero:

$$
a_{n}, b_{n} \in \mathbb{C}, \quad a_{n} \neq 0
$$

In the real case

$$
a_{n}, b_{n} \in \mathbb{R}, \quad a_{n} \neq 0,
$$

the matrix $J$ is Hermitian (self-adjoint) and in this case many versions of the inverse spectral problem for $J$ have been investigated in the literature, see $[1,2,3]$ and references given therein.

In the complex case (1.2), the matrix $J$ is in general non-Hermitian (non-selfadjoint) and our aim in this paper is to introduce appropriate spectral data for such a matrix and then consider the inverse spectral problem consisting in determining of the matrix from its spectral data.

${ }^{\star}$ This paper is a contribution to the Proceedings of the VIIth Workshop "Quantum Physics with NonHermitian Operators" (June 29 - July 11, 2008, Benasque, Spain). The full collection is available at http://www.emis.de/journals/SIGMA/PHHQP2008.html 
As is known $[4,5,6,7,8,9]$, for non-selfadjoint differential and difference operators a natural spectral characteristic is the so-called generalized spectral function which is a linear continuous functional on an appropriate linear topological space. In general very little is known about the structure of generalized spectral functions.

Given the matrix $J$ of the form (1.1) with the entries satisfying (1.2), consider the eigenvalue problem $J y=\lambda y$ for a column vector $y=\left\{y_{n}\right\}_{n=0}^{N-1}$, that is equivalent to the second order linear difference equation

$$
a_{n-1} y_{n-1}+b_{n} y_{n}+a_{n} y_{n+1}=\lambda y_{n}, \quad n \in\{0,1, \ldots, N-1\}, \quad a_{-1}=a_{N-1}=1,
$$

for $\left\{y_{n}\right\}_{n=-1}^{N}$, with the boundary conditions

$$
y_{-1}=y_{N}=0 \text {. }
$$

The problem (1.4), (1.5) is a discrete analogue of the continuous eigenvalue value problem

$$
\begin{aligned}
& \frac{d}{d x}\left[p(x) \frac{d}{d x} y(x)\right]+q(x) y(x)=\lambda y(x), \quad x \in[a, b], \\
& y(a)=y(b)=0
\end{aligned}
$$

where $[a, b]$ is a finite interval.

To the continuous problem

$$
\begin{aligned}
& \frac{d}{d x}\left[p(x) \frac{d}{d x} y(x)\right]+q(x) y(x)=\lambda y(x), \quad x \in[0, \infty), \\
& y(0)=0
\end{aligned}
$$

on the semi-infinite interval $[0, \infty)$ there corresponds a Jacobi matrix of the type (1.1) but $J$ being infinite both downwards and to the right. To the equation in (1.6) considered on the whole real axis $(-\infty, \infty)$ there corresponds a Jacobi matrix which is infinite in the all four directions: upwards, downwards, to the left, and to the right.

The case of infinite Jacobi matrices was considered earlier in the papers $[6,7,8,9]$ in which the generalized spectral function was introduced and the inverse problem from the generalized spectral function was studied. However, in the case of infinite Jacobi matrices the structure of the generalized spectral function does not allow any explicit description because of complexity of the structure. Our main achievement in the present paper is that we describe explicitly the structure of the generalized spectral function for the finite order Jacobi matrices (1.1), (1.2).

The paper is organized as follows. In Section 2, the generalized spectral function is introduced for Jacobi matrices of the form (1.1) with the entries satisfying (1.2). In Section 3, the inverse problem from the generalized spectral function is investigated. It turns out that the matrix (1.1) is not uniquely restored from the generalized spectral function. There are precisely $2^{N-1}$ distinct Jacobi matrices possessing the same generalized spectral function. The inverse problem is solved uniquely from the data consisting of the generalized spectral function and a sequence $\left\{\sigma_{1}, \sigma_{2}, \ldots, \sigma_{N-1}\right\}$ of signs + and - . Section 4 is devoted to some examples. In Section 5 , we describe the structure of the generalized spectral function and in this way we define the concept of spectral data for matrices (1.1). In Section 6, the inverse problem from the spectral data is considered. In Section 7, we characterize generalized spectral functions of real Jacobi matrices among the generalized spectral functions of complex Jacobi matrices. In Section 8, we describe the structure of generalized spectral functions and spectral data of real Jacobi matrices. Finally, in Section 9, we consider inverse problem for real Jacobi matrices from the spectral data.

Note that considerations of complex (non-Hermitian) Hamiltonians in quantum mechanics and complex discrete models have recently received a lot of attention [10, 11, 12, 13]. For some 
recent papers dealing with the spectral theory of difference (and differential) operators with complex coefficients see $[14,15,16,17,18]$. Fur further reading on the spectral theory of the Jacobi difference equation (three term recurrence relation) the books [19, 20, 21, 22, 23] are excellent sources.

\section{Generalized spectral function}

Given a matrix $J$ of the form (1.1) with the entries satisfying (1.2). Consider the eigenvalue problem $J y=\lambda y$ for a column vector $y=\left\{y_{n}\right\}_{n=0}^{N-1}$, that is equivalent to the second order linear difference equation

$$
a_{n-1} y_{n-1}+b_{n} y_{n}+a_{n} y_{n+1}=\lambda y_{n}, \quad n \in\{0,1, \ldots, N-1\}, \quad a_{-1}=a_{N-1}=1,
$$

for $\left\{y_{n}\right\}_{n=-1}^{N}$, with the boundary conditions

$$
y_{-1}=y_{N}=0 \text {. }
$$

Denote by $\left\{P_{n}(\lambda)\right\}_{n=-1}^{N}$ the solution of equation (2.1) satisfying the initial conditions

$$
y_{-1}=0, \quad y_{0}=1 \text {. }
$$

Using (2.3), we can find from equation (2.1) recurrently the quantities $P_{n}(\lambda)$ for $n=1,2, \ldots, N$; $P_{n}(\lambda)$ is a polynomial in $\lambda$ of degree $n$.

Thus $\left\{P_{n}(\lambda)\right\}_{n=0}^{N}$ is the unique solution of the recursion relations

$$
\begin{aligned}
& b_{0} P_{0}(\lambda)+a_{0} P_{1}(\lambda)=\lambda P_{0}(\lambda), \\
& a_{n-1} P_{n-1}(\lambda)+b_{n} P_{n}(\lambda)+a_{n} P_{n+1}(\lambda)=\lambda P_{n}(\lambda), \\
& n \in\{1,2, \ldots, N-1\}, \quad a_{N-1}=1,
\end{aligned}
$$

subject to the initial condition

$$
P_{0}(\lambda)=1 \text {. }
$$

Lemma 1. The equality

$$
\operatorname{det}(J-\lambda I)=(-1)^{N} a_{0} a_{1} \cdots a_{N-2} P_{N}(\lambda)
$$

holds so that the eigenvalues of the matrix $J$ coincide with the zeros of the polynomial $P_{N}(\lambda)$.

Proof. To prove (2.6) let us set, for each $n \in\{1,2, \ldots, N\}$,

$$
J_{n}=\left[\begin{array}{ccccccc}
b_{0} & a_{0} & 0 & \cdots & 0 & 0 & 0 \\
a_{0} & b_{1} & a_{1} & \cdots & 0 & 0 & 0 \\
0 & a_{1} & b_{2} & \cdots & 0 & 0 & 0 \\
\vdots & \vdots & \vdots & \ddots & \vdots & \vdots & \vdots \\
0 & 0 & 0 & \cdots & b_{n-3} & a_{n-3} & 0 \\
0 & 0 & 0 & \cdots & a_{n-3} & b_{n-2} & a_{n-2} \\
0 & 0 & 0 & \cdots & 0 & a_{n-2} & b_{n-1}
\end{array}\right]
$$

and set $\Delta_{n}(\lambda)=\operatorname{det}\left(J_{n}-\lambda I\right)$. Note that by $I$ we denote an identity matrix of needed size. By expanding the determinant $\operatorname{det}\left(J_{n+1}-\lambda I\right)$ by the elements of the last row, it is easy to show that

$$
\Delta_{n+1}(\lambda)=\left(b_{n}-\lambda\right) \Delta_{n}(\lambda)-a_{n-1}^{2} \Delta_{n-1}(\lambda), \quad n=1,2, \ldots ; \quad \Delta_{0}(\lambda)=1 .
$$


Dividing this equation by the product $a_{0} \cdots a_{n-1}$, we find that the sequence

$$
d_{-1}=0, d_{0}=1, d_{n}=(-1)^{n}\left(a_{0} \cdots a_{n-1}\right)^{-1} \Delta_{n}(\lambda), \quad n=1,2, \ldots,
$$

satisfies (2.1), (2.3). Then $d_{n}=P_{n}(\lambda), n=0,1, \ldots$, and hence we have (2.6) because $J_{N}=J$ and $a_{N-1}=1$.

For any nonnegative integer $m$ denote by $\mathbb{C}_{m}[\lambda]$ the ring of all polynomials in $\lambda$ of degree $\leq m$ with complex coefficients. A mapping $\Omega: \mathbb{C}_{m}[\lambda] \rightarrow \mathbb{C}$ is called a linear functional if for any $G(\lambda), H(\lambda) \in \mathbb{C}_{m}[\lambda]$ and $\alpha \in \mathbb{C}$, we have

$$
\langle\Omega, G(\lambda)+H(\lambda)\rangle=\langle\Omega, G(\lambda)\rangle+\langle\Omega, H(\lambda)\rangle \quad \text { and } \quad\langle\Omega, \alpha G(\lambda)\rangle=\alpha\langle\Omega, G(\lambda)\rangle,
$$

where $\langle\Omega, G(\lambda)\rangle$ denotes the value of $\Omega$ on the element (polynomial) $G(\lambda)$.

Theorem 1. There exists a unique linear functional $\Omega: \mathbb{C}_{2 N}[\lambda] \rightarrow \mathbb{C}$ such that the relations

$$
\begin{aligned}
& \left\langle\Omega, P_{m}(\lambda) P_{n}(\lambda)\right\rangle=\delta_{m n}, \quad m, n \in\{0,1, \ldots, N-1\}, \\
& \left\langle\Omega, P_{m}(\lambda) P_{N}(\lambda)\right\rangle=0, \quad m \in\{0,1, \ldots, N\},
\end{aligned}
$$

hold, where $\delta_{m n}$ is the Kronecker delta.

Proof. First we prove the uniqueness of $\Omega$. Assume that there exists a linear functional $\Omega$ possessing the properties (2.7) and (2.8). The $2 N+1$ polynomials

$$
P_{n}(\lambda) \quad(n=0,1, \ldots, N-1), \quad P_{m}(\lambda) P_{N}(\lambda) \quad(m=0,1, \ldots, N)
$$

form a basis for the linear space $\mathbb{C}_{2 N}[\lambda]$ because they are linearly independent (their degrees are distinct) and their number $2 N+1=\operatorname{dim} \mathbb{C}_{2 N}[\lambda]$. On the other hand, by (2.7) and (2.8) the functional $\Omega$ takes on polynomials (2.9) completely definite values:

$$
\begin{aligned}
& \left\langle\Omega, P_{n}(\lambda)\right\rangle=\delta_{0 n}, \quad n \in\{0,1, \ldots, N-1\}, \\
& \left\langle\Omega, P_{m}(\lambda) P_{N}(\lambda)\right\rangle=0, \quad m \in\{0,1, \ldots, N\} .
\end{aligned}
$$

Therefore $\Omega$ is determined on $\mathbb{C}_{2 N}[\lambda]$ uniquely by the linearity.

To prove existence of $\Omega$ we define it on the basis polynomials (2.9) by (2.10), (2.11) and then we extend $\Omega$ to over the whole space $\mathbb{C}_{2 N}[\lambda]$ by linearity. Let us show that the functional $\Omega$ defined in this way satisfies (2.7), (2.8). Denote

$$
\left\langle\Omega, P_{m}(\lambda) P_{n}(\lambda)\right\rangle=A_{m n}, \quad m, n \in\{0,1, \ldots, N\} .
$$

Obviously, $A_{m n}=A_{n m}$ for $m, n \in\{0,1, \ldots, N\}$. From (2.10) and (2.11) we have

$$
\begin{aligned}
& A_{m 0}=A_{0 m}=\delta_{m 0}, \quad m \in\{0,1, \ldots, N\}, \\
& A_{m N}=A_{N m}=0, \quad m \in\{0,1, \ldots, N\} .
\end{aligned}
$$

Since $\left\{P_{n}(\lambda)\right\}_{0}^{N}$ is the solution of equations (2.4), we find from the first equation, recalling (2.5),

$$
\lambda=b_{0}+a_{0} P_{1}(\lambda) .
$$

Substituting this in the remaining equations of (2.4), we obtain

$$
\begin{aligned}
& a_{n-1} P_{n-1}(\lambda)+b_{n} P_{n}(\lambda)+a_{n} P_{n+1}(\lambda)=b_{0} P_{n}(\lambda)+a_{0} P_{1}(\lambda) P_{n}(\lambda), \\
& n \in\{1,2, \ldots, N-1\}, \quad a_{N-1}=1 .
\end{aligned}
$$


Applying the functional $\Omega$ to both sides of the last equation, and recalling (2.13) and (2.14), we get

$$
A_{n 1}=A_{1 n}=\delta_{n 1}, \quad n \in\{0,1, \ldots, N\} .
$$

Further, since

$$
\begin{aligned}
& a_{m-1} P_{m-1}(\lambda)+b_{m} P_{m}(\lambda)+a_{m} P_{m+1}(\lambda)=\lambda P_{m}(\lambda), \quad m \in\{1,2, \ldots, N-1\}, \\
& a_{n-1} P_{n-1}(\lambda)+b_{n} P_{n}(\lambda)+a_{n} P_{n+1}(\lambda)=\lambda P_{n}(\lambda), \quad n \in\{1,2, \ldots, N-1\},
\end{aligned}
$$

we obtain, multiplying the first of these identities by $P_{n}(\lambda)$, and multiplying the second by $P_{m}(\lambda)$, then subtracting the second result from the first:

$$
\begin{aligned}
& a_{m-1} P_{m-1}(\lambda) P_{n}(\lambda)+b_{m} P_{m}(\lambda) P_{n}(\lambda)+a_{m} P_{m+1}(\lambda) P_{n}(\lambda) \\
& \quad=a_{n-1} P_{n-1}(\lambda) P_{m}(\lambda)+b_{n} P_{n}(\lambda) P_{m}(\lambda)+a_{n} P_{n+1}(\lambda) P_{m}(\lambda), \quad m, n \in\{1,2, \ldots, N-1\} .
\end{aligned}
$$

Applying the functional $\Omega$ to both sides of the last equation, and recalling (2.13), (2.14), and (2.15), we obtain for $A_{m n}$ the boundary value problem

$$
\begin{aligned}
& a_{m-1} A_{m-1, n}+b_{m} A_{m n}+a_{m} A_{m+1, n}=a_{n-1} A_{n-1, m}+b_{n} A_{n m}+a_{n} A_{n+1, m}, \\
& \quad m, n \in\{1,2, \ldots, N-1\} \\
& A_{n 0}=A_{0 n}=\delta_{n 0}, \quad A_{n 1}=A_{1 n}=\delta_{n 1}, \quad A_{N n}=A_{n N}=0 \\
& \quad n \in\{0,1, \ldots, N\} .
\end{aligned}
$$

Using(2.17), we can find from (2.16) recurrently all the $A_{m n}$ and the unique solution of problem (2.16), (2.17) is $A_{m n}=\delta_{m n}$ for $m, n \in\{0,1, \ldots, N-1\}$ and $A_{m N}=0$ for $m \in\{0,1, \ldots, N\}$.

Definition 1. The linear functional $\Omega$ defined in Theorem 1 we call the generalized spectral function of the matrix $J$ given in (1.1).

\section{Inverse problem from the generalized spectral function}

The inverse problem is stated as follows:

1. To see if it is possible to reconstruct the matrix $J$, given its generalized spectral function $\Omega$. If it is possible, to describe the reconstruction procedure.

2. To find the necessary and sufficient conditions for a given linear functional $\Omega$ on $\mathbb{C}_{2 N}[\lambda]$, to be the generalized spectral function for some matrix $J$ of the form (1.1) with entries belonging to the class (1.2).

Since $P_{n}(\lambda)$ is a polynomial of degree $n$, we can write the representation

$$
P_{n}(\lambda)=\alpha_{n}\left(\lambda^{n}+\sum_{k=0}^{n-1} \chi_{n k} \lambda^{k}\right), \quad n \in\{0,1, \ldots, N\} .
$$

Substituting (3.1) in (2.4), we find that the coefficients $a_{n}, b_{n}$ of system (2.4) and the quantities $\alpha_{n}, \chi_{n k}$ of decomposition (3.1), are interconnected by the equations

$$
\begin{aligned}
& a_{n}=\frac{\alpha_{n}}{\alpha_{n+1}} \quad(0 \leq n \leq N-2), \quad \alpha_{0}=1, \quad \alpha_{N}=\alpha_{N-1}, \\
& b_{n}=\chi_{n, n-1}-\chi_{n+1, n} \quad(0 \leq n \leq N-1), \quad \chi_{0,-1}=0 .
\end{aligned}
$$


It is easily seen that relations $(2.7),(2.8)$ are equivalent to the collection of the relations

$$
\begin{aligned}
& \left\langle\Omega, \lambda^{m} P_{n}(\lambda)\right\rangle=\frac{\delta_{m n}}{\alpha_{n}}, \quad m=0,1, \ldots, n, \quad n \in\{0,1, \ldots, N-1\}, \\
& \left\langle\Omega, \lambda^{m} P_{N}(\lambda)\right\rangle=0, \quad m=0,1, \ldots, N .
\end{aligned}
$$

In fact, using (3.1), we have

$$
\left\langle\Omega, P_{m}(\lambda) P_{n}(\lambda)\right\rangle=\alpha_{m}\left\langle\Omega, \lambda^{m} P_{n}(\lambda)\right\rangle+\alpha_{m} \sum_{j=0}^{m-1} \chi_{m j}\left\langle\Omega, \lambda^{j} P_{n}(\lambda)\right\rangle .
$$

Next, since we have the expansion

$$
\lambda^{j}=\sum_{i=0}^{j} c_{i}^{(j)} P_{i}(\lambda), \quad j \in\{0,1, \ldots, N\},
$$

it follows from (3.6) that (3.4), (3.5) hold if we have (2.7), (2.8). The converse is also true: if (3.4), (3.5) hold, then (2.7), (2.8) can be obtained from (3.6), in conjunction with (3.1).

Let us set

$$
s_{l}=\left\langle\Omega, \lambda^{l}\right\rangle, \quad l \in\{0,1, \ldots, 2 N\},
$$

that are the "power moments" of the functional $\Omega$.

Replacing $P_{n}(\lambda)$ and $P_{N}(\lambda)$ in (3.4) and (3.5) by their expansions in (3.1), we obtain

$$
\begin{array}{ll}
s_{n+m}+\sum_{k=0}^{n-1} \chi_{n k} s_{k+m}=0, & m=0,1, \ldots, n-1, \quad n \in\{1,2, \ldots, N\}, \\
s_{2 N}+\sum_{k=0}^{N-1} \chi_{N k} s_{k+N}=0, & \\
s_{2 n}+\sum_{k=0}^{n-1} \chi_{n k} s_{k+n}=\frac{1}{\alpha_{n}^{2}}, \quad n \in\{0,1, \ldots, N-1\} . &
\end{array}
$$

Notice that (3.8) is the fundamental equation of the inverse problem, in the sense that it enables the problem to be formally solved. For, if we are given the linear functional $\Omega$ on $\mathbb{C}_{2 N}[\lambda]$, we can find the quantities $s_{l}$ from (3.7) and then we consider the inhomogeneous system of linear algebraic equations (3.8) with unknowns $\chi_{n 0}, \chi_{n 1}, \ldots, \chi_{n, n-1}$, for every fixed $n \in\{1,2, \ldots, N\}$. If this system is uniquely solvable, and $s_{2 n}+\sum_{k=0}^{n-1} \chi_{n k} s_{k+n} \neq 0$ for $n \in\{1,2, \ldots, N-1\}$, then the entries $a_{n}, b_{n}$ of the required matrix $J$ can be found from (3.2) and (3.3), respectively, $\alpha_{n}$ being found from (3.10). The next theorem gives the conditions under which the indicated procedure of solving the inverse problem is rigorously justified.

Theorem 2. In order for a given linear functional $\Omega$, defined on $\mathbb{C}_{2 N}[\lambda]$, to be the generalized spectral function for some Jacobi matrix $J$ of the form (1.1) with entries belonging to the class (1.2), it is necessary and sufficient that the following conditions be satisfied:

(i) $\langle\Omega, 1\rangle=1$ (normalization condition);

(ii) if, for some polynomial $G(\lambda), \operatorname{deg} G(\lambda) \leq N-1$,

$$
\langle\Omega, G(\lambda) H(\lambda)\rangle=0
$$

for all polynomials $H(\lambda), \operatorname{deg} H(\lambda)=\operatorname{deg} G(\lambda)$, then $G(\lambda) \equiv 0$; 
(iii) there exists a polynomial $T(\lambda)$ of degree $N$ such that

$$
\langle\Omega, G(\lambda) T(\lambda)\rangle=0
$$

for all polynomials $G(\lambda)$ with $\operatorname{deg} G(\lambda) \leq N$.

Proof. Necessity. We obtain (i) from (2.7) with $n=m=0$, recalling (2.5). To prove (ii), we write the expansion

$$
G(\lambda)=\sum_{j=0}^{m} c_{j}^{(m)} P_{j}(\lambda), \quad m=\operatorname{deg} G(\lambda),
$$

and take as $H(\lambda)$ the polynomial

$$
H(\lambda)=\sum_{j=0}^{m} \overline{c_{j}^{(m)}} P_{j}(\lambda)
$$

where the bar over a complex number denotes the complex conjugation. Then we find from $\langle\Omega, G(\lambda) H(\lambda)\rangle=0$ using $(2.7)$ that

$$
\sum_{j=0}^{m}\left|c_{j}^{(m)}\right|^{2}=0
$$

hence $c_{j}^{(m)}=0, j=0,1, \ldots, m$, i.e., $G(\lambda) \equiv 0$. The statement (iii) of the theorem follows from (2.8) if we take $T(\lambda)=P_{N}(\lambda)$.

Sufficiency. The proof will be given in several stages.

(a) Given the linear functional $\Omega$, defined on $\mathbb{C}_{2 N}[\lambda]$ and satisfying the conditions of the theorem. Consider equation (3.8) with the unknowns $\chi_{n k}, k=0,1, \ldots, n-1$, in which $s_{l}$ are found with the aid of the functional $\Omega$ from expression (3.7). Let us show that this equation has a unique solution for every fixed $n \in\{1,2, \ldots, N\}$. For this, it is sufficient to show that the corresponding homogeneous equation

$$
\sum_{k=0}^{n-1} g_{k} s_{k+m}=0, \quad m=0,1, \ldots, n-1,
$$

has only the zero solution for every $n$. Assume the contrary. For some $n \in\{1,2, \ldots, N\}$ let equation (3.11) have the nonzero solution $\left(g_{k}\right)_{0}^{n-1}$. Further let $\left(h_{m}\right)_{0}^{n-1}$ be an arbitrary vector. We multiply both sides of (3.11) by $h_{m}$ and sum over $m$ between 0 and $n-1$; we get

$$
\sum_{m=0}^{n-1} \sum_{k=0}^{n-1} h_{m} g_{k} s_{k+m}=0 .
$$

Substituting expression (3.7) for $s_{k+m}$ in this equation and denoting

$$
G(\lambda)=\sum_{k=0}^{n-1} g_{k} \lambda^{k}, \quad H(\lambda)=\sum_{m=0}^{n-1} h_{m} \lambda^{m},
$$

we obtain

$$
\langle\Omega, G(\lambda) H(\lambda)\rangle=0 .
$$


Since $\left(h_{m}\right)_{0}^{n-1}$ is an arbitrary vector, we find from (3.12), in the light of condition (ii) of the theorem, that $G(\lambda) \equiv 0$, and hence $g_{0}=g_{1}=\cdots=g_{n-1}=0$, in spite of our assumption. Thus, for any $n \in\{1,2, \ldots, N\}$, equation (3.8) has a unique solution.

(b) Let us show that

$$
s_{2 n}+\sum_{k=0}^{n-1} \chi_{n k} s_{k+n} \neq 0, \quad n \in\{1,2, \ldots, N-1\},
$$

where $\left(\chi_{n k}\right)_{k=0}^{n-1}$ is the solution of the fundamental equation (3.8). (For $n=0$, the left-hand side of $(3.13)$ is $s_{0}=\langle\Omega, 1\rangle=1$.) Assume the contrary, i.e., for some $n \in\{1,2, \ldots, N-1\}$

$$
s_{2 n}+\sum_{k=0}^{n-1} \chi_{n k} s_{k+n}=0
$$

Joining this equation to the fundamental equation (3.8), we obtain

$$
s_{n+m}+\sum_{k=0}^{n-1} \chi_{n k} s_{k+m}=0, \quad m=0,1, \ldots, n .
$$

Let $\left(h_{m}\right)_{0}^{n}$ be an arbitrary vector. Multiplying both sides of (3.14) by $h_{m}$ and summing over $m$ from 0 to $n$, we obtain

$$
\sum_{m=0}^{n} h_{m} s_{n+m}+\sum_{m=0}^{n} \sum_{k=0}^{n-1} h_{m} \chi_{n k} s_{k+m}=0
$$

Replacing $s_{l}$ in this by its expression (3.7), we obtain

$$
\left\langle\Omega,\left[\lambda^{n}+\chi(\lambda)\right] H(\lambda)\right\rangle=0,
$$

where

$$
\chi(\lambda)=\sum_{k=0}^{n-1} \chi_{n k} \lambda^{k}, \quad H(\lambda)=\sum_{m=0}^{n} h_{m} \lambda^{m} .
$$

Since $\left(h_{m}\right)_{0}^{n}$ is an arbitrary vector, we obtain from the last equation in the light of condition (ii) of the theorem:

$$
\lambda^{n}+\chi(\lambda) \equiv 0
$$

which is impossible. Our assumption is therefore false.

(c) Given the solution $\left(\chi_{n k}\right)_{k=0}^{n-1}$ of the fundamental equation (3.8), we find $\alpha_{n}$ from (3.10) for $n \in\{0,1, \ldots, N-1\}$ with $\alpha_{0}=1$ and set $\alpha_{N}=\alpha_{N-1}$. Then we find the polynomials $P_{n}(\lambda)$ from (3.1). Let us show that the relations (2.7), (2.8) hold. It is enough to show that (3.4), (3.5) hold because (3.4), (3.5) together are equivalent to relations (2.7), (2.8). From (3.8) and (3.10) we have (3.4) and (3.5), the latter except for $m=N$. So it remains to show that

$$
\left\langle\Omega, \lambda^{N} P_{N}(\lambda)\right\rangle=0 .
$$

For this purpose we use the condition (iii) of the theorem. By this condition we have

$$
T(\lambda)=\sum_{k=0}^{N} t_{k} \lambda^{k}, \quad t_{N} \neq 0,
$$


and

$$
0=\left\langle\Omega, P_{N}(\lambda) T(\lambda)\right\rangle=\sum_{k=0}^{N} t_{k}\left\langle\Omega, \lambda^{k} P_{N}(\lambda)\right\rangle=t_{N}\left\langle\Omega, \lambda^{N} P_{N}(\lambda)\right\rangle,
$$

where we have used (3.5) except for $m=N$. Hence $\left\langle\Omega, \lambda^{N} P_{N}(\lambda)\right\rangle=0$.

(d) Let us show that the polynomials $P_{n}(\lambda), n=0,1, \ldots, N$, constructed in accordance with (3.1) with the aid of the numbers $\chi_{n k}$ and $\alpha_{n}$ obtained above, satisfy the equations

$$
\begin{aligned}
& b_{0} P_{0}(\lambda)+a_{0} P_{1}(\lambda)=\lambda P_{0}(\lambda), \\
& a_{n-1} P_{n-1}(\lambda)+b_{n} P_{n}(\lambda)+a_{n} P_{n+1}(\lambda)=\lambda P_{n}(\lambda), \\
& n \in\{1,2, \ldots, N-1\}, \quad a_{N-1}=1,
\end{aligned}
$$

where the coefficients $a_{n}, b_{n}$ are given by the expressions

$$
\begin{aligned}
& a_{n}=\frac{\alpha_{n}}{\alpha_{n+1}} \quad(0 \leq n \leq N-2), \quad \alpha_{0}=1, \quad \alpha_{N}=\alpha_{N-1}, \\
& b_{n}=\chi_{n, n-1}-\chi_{n+1, n} \quad(0 \leq n \leq N-1), \quad \chi_{0,-1}=0 .
\end{aligned}
$$

We first verify the first equation of (3.15). From (3.1) we have

$$
P_{0}(\lambda)=1, \quad P_{1}(\lambda)=\alpha_{1}\left(\lambda+\chi_{10}\right) .
$$

Hence the first equation of (3.15) has the form

$$
b_{0}+a_{0} \alpha_{1}\left(\lambda+\chi_{10}\right)=\lambda
$$

and this is true, since, by (3.16) and (3.17),

$$
a_{0} \alpha_{1}=1, \quad b_{0}=-\chi_{10}
$$

Let us prove the remaining equations of (3.15). Since $\lambda P_{n}(\lambda)$ is a polynomial of degree $n+1$, while $P_{k}(\lambda), k=0,1, \ldots, n+1$, are linearly independent, we have

$$
\lambda P_{n}(\lambda)=\sum_{k=0}^{n+1} c_{k}^{(n)} P_{k}(\lambda) \quad n \in\{1,2, \ldots, N-1\}, \quad c_{N}^{(N-1)}=1,
$$

where $c_{k}^{(n)}, k=0,1, \ldots, n+1$, are constants. By (2.7), (2.8) which we proved in (c), we have from (3.18):

$$
c_{k}^{(n)}=\left\langle\Omega, \lambda P_{n}(\lambda) P_{k}(\lambda)\right\rangle, \quad k=0,1, \ldots, n+1 \quad(n \in\{1,2, \ldots, N-2\}) .
$$

The polynomials $\lambda P_{k}(\lambda), k=0,1, \ldots, n-2$, have degrees $\leq n-1$, and hence we find from (3.19) in the light of (2.7), (2.8) that

$$
c_{k}^{(n)}=0, \quad k=0,1, \ldots, n-2 \quad(n \in\{1,2, \ldots, N-1\}) .
$$

Consequently, expansion (3.18) takes the form

$$
c_{n-1}^{(n)} P_{n-1}(\lambda)+c_{n}^{(n)} P_{n}(\lambda)+c_{n+1}^{(n)} P_{n+1}(\lambda)=\lambda P_{n}(\lambda), \quad n \in\{1,2, \ldots, N-1\} .
$$

It follows from (3.19) that $c_{n-1}^{(n)}=c_{n}^{(n-1)}$. Hence, denoting

$$
c_{n+1}^{(n)}=\widetilde{a}_{n}, \quad c_{n}^{(n)}=\widetilde{b}_{n},
$$


we have from (3.20):

$$
\widetilde{a}_{n-1} P_{n-1}(\lambda)+\widetilde{b}_{n} P_{n}(\lambda)+\widetilde{a}_{n} P_{n+1}(\lambda)=\lambda P_{n}(\lambda), \quad n \in\{1,2, \ldots, N-1\} .
$$

Replacing $P_{n}(\lambda)$ in (3.22) by its expression (3.1) and equating coefficients of like $\lambda^{n}$, while recalling (3.16), (3.17), we obtain

$$
\begin{aligned}
& \widetilde{a}_{n}=\frac{\alpha_{n}}{\alpha_{n+1}}=a_{n} \quad(0 \leq n \leq N-2), \\
& \widetilde{b}_{n}=\chi_{n, n-1}-\chi_{n+1, n}=b_{n} \quad(0 \leq n \leq N-1) .
\end{aligned}
$$

Theorem 2 is completely proved.

Remark 1. It follows from the above solution of the inverse problem that the matrix (1.1) is not uniquely restored from the generalized spectral function. This is linked with the fact that the $\alpha_{n}$ are determined from (3.10) uniquely up to a sign. To ensure that the inverse problem is uniquely solvable, we have to specify additionally a sequence of signs + and - . Namely, let $\left\{\sigma_{1}, \sigma_{2}, \ldots, \sigma_{N-1}\right\}$ be a given finite sequence, where for each $n \in\{1,2, \ldots, N-1\}$ the $\sigma_{n}$ is + or - . We have $2^{N-1}$ such different sequences. Now to determine $\alpha_{n}$ uniquely from (3.10) for $n \in\{1,2, \ldots, N-1\}$ (remember that we always take $\alpha_{0}=1$ ) we can choose the sign $\sigma_{n}$ when extracting the square root. In this way we get precisely $2^{N-1}$ distinct Jacobi matrices possessing the same generalized spectral function. For example, the two matrices

$$
\left[\begin{array}{ll}
1 & 1 \\
1 & 1
\end{array}\right], \quad\left[\begin{array}{cc}
1 & -1 \\
-1 & 1
\end{array}\right]
$$

as well as the four matrices

$$
\left[\begin{array}{lll}
1 & 1 & 0 \\
1 & 1 & 1 \\
0 & 1 & 1
\end{array}\right], \quad\left[\begin{array}{ccc}
1 & -1 & 0 \\
-1 & 1 & 1 \\
0 & 1 & 1
\end{array}\right], \quad\left[\begin{array}{ccc}
1 & 1 & 0 \\
1 & 1 & -1 \\
0 & -1 & 1
\end{array}\right], \quad\left[\begin{array}{ccc}
1 & -1 & 0 \\
-1 & 1 & -1 \\
0 & -1 & 1
\end{array}\right],
$$

have the same generalized spectral function. The inverse problem is solved uniquely from the data consisting of $\Omega$ and a sequence $\left\{\sigma_{1}, \sigma_{2}, \ldots, \sigma_{N-1}\right\}$ of signs + and -.

Using the numbers

$$
s_{l}=\left\langle\Omega, \lambda^{l}\right\rangle, \quad l=0,1, \ldots, 2 N,
$$

let us introduce the determinants

$$
D_{n}=\left|\begin{array}{cccc}
s_{0} & s_{1} & \cdots & s_{n} \\
s_{1} & s_{2} & \cdots & s_{n+1} \\
\vdots & \vdots & \ddots & \vdots \\
s_{n} & s_{n+1} & \cdots & s_{2 n}
\end{array}\right|, \quad n=0,1, \ldots, N
$$

It turns out that Theorem 2 is equivalent to the following theorem.

Theorem 3. In order for a given linear functional $\Omega$, defined on $\mathbb{C}_{2 N}[\lambda]$, to be the generalized spectral function for some Jacobi matrix $J$ of the form (1.1) with entries belonging to the class (1.2), it is necessary and sufficient that

$$
D_{0}=1, \quad D_{n} \neq 0 \quad(n=1,2, \ldots, N-1), \quad \text { and } \quad D_{N}=0,
$$

where $D_{n}$ is defined by (3.24) and (3.23). 
Proof. Necessity. The condition $D_{0}=1$ follows from $1=\langle\Omega, 1\rangle=s_{0}=D_{0}$. By Theorem 2, if for a polynomial

$$
G(\lambda)=\sum_{k=0}^{n} g_{k} \lambda^{k}
$$

with $n \leq N-1$ we have

$$
\langle\Omega, G(\lambda) H(\lambda)\rangle=0
$$

for all polynomials

$$
H(\lambda)=\sum_{m=0}^{n} h_{m} \lambda^{m},
$$

then $G(\lambda) \equiv 0$, that is, $g_{0}=g_{1}=\cdots=g_{n}=0$.

If we substitute (3.26) and (3.28) in (3.27), then we get

$$
\sum_{m=0}^{n} h_{m}\left(\sum_{k=0}^{n} g_{k} s_{k+m}\right)=0 .
$$

Since $h_{0}, h_{1}, \ldots, h_{n}$ are arbitrary, the last equation gives

$$
\sum_{k=0}^{n} g_{k} s_{k+m}=0, \quad m=0,1, \ldots, n .
$$

This is a linear homogeneous system of algebraic equations with respect to $g_{0}, g_{1}, \ldots, g_{n}$ and the determinant of this system coincides with the determinant $D_{n}$. Since this system has only the trivial solution $g_{0}=g_{1}=\cdots=g_{n}=0$, we have that $D_{n} \neq 0$, where $n \leq N-1$.

To prove that $D_{N}=0$, we write equation (3.8) for $n=N$ to get

$$
s_{N+m}+\sum_{k=0}^{N-1} \chi_{N k} s_{k+m}=0, \quad m=0,1, \ldots, N-1 .
$$

This equation has the unique solution $\chi_{N 0}, \chi_{N 1}, \ldots, \chi_{N, N-1}$. Next, these equalities together with (3.9) can be written in the form

$$
\left[\begin{array}{c}
s_{N} \\
s_{N+1} \\
\vdots \\
s_{2 N-1} \\
s_{2 N}
\end{array}\right]+\chi_{N 0}\left[\begin{array}{c}
s_{0} \\
s_{1} \\
\vdots \\
s_{N-1} \\
s_{N}
\end{array}\right]+\chi_{N 1}\left[\begin{array}{c}
s_{1} \\
s_{2} \\
\vdots \\
s_{N} \\
s_{N+1}
\end{array}\right]+\cdots+\chi_{N, N-1}\left[\begin{array}{c}
s_{N-1} \\
s_{N} \\
\vdots \\
s_{2 N-2} \\
s_{2 N-1}
\end{array}\right]=0
$$

This means that the last column in the determinant $D_{N}$ is a linear combination of the remaining columns. Therefore $D_{N}=0$.

Sufficiency. Given the linear functional $\Omega: \mathbb{C}_{2 N}[\lambda] \rightarrow \mathbb{C}$ satisfying the conditions (3.25), it is enough to show that then the conditions of Theorem 2 are satisfied. We have $\langle\Omega, 1\rangle=s_{0}=$ $D_{0}=1$. Next, let (3.27) hold for a polynomial $G(\lambda)$ of the form (3.26) and all polynomials $H(\lambda)$ of the form (3.28). Then (3.29) holds. Since the determinant of this system is $D_{n}$ and $D_{n} \neq 0$ for $n \leq N-1$, we get that $g_{0}=g_{1}=\cdots=g_{n}=0$, that is, $G(\lambda) \equiv 0$. Finally, we have to show that there is a polynomial $T(\lambda)$ of degree $N$ such that

$$
\langle\Omega, G(\lambda) T(\lambda)\rangle=0
$$


for all polynomials $G(\lambda)$ with $\operatorname{deg} G(\lambda) \leq N$. For this purpose we consider the homogeneous system

$$
\sum_{k=0}^{N} t_{k} s_{k+m}=0, \quad m=0,1, \ldots, N,
$$

with the unknowns $t_{0}, t_{1}, \ldots, t_{N}$. The determinant of this system is $D_{N}$. Since by condition $D_{N}=0$, this system has a nontrivial solution $t_{0}, t_{1}, \ldots, t_{N}$. We have $t_{N} \neq 0$. Indeed, if $t_{N}=0$, then we get from (3.31)

$$
\sum_{k=0}^{N-1} t_{k} s_{k+m}=0, \quad m=0,1, \ldots, N-1 .
$$

The determinant of this system is $D_{N-1}$ and by condition $D_{N-1} \neq 0$. Then $t_{0}=t_{1}=\cdots=$ $t_{N-1}=0$ and we get that the solution $t_{0}, t_{1}, \ldots, t_{N}$ of system (3.31) is trivial, which is a contradiction. Taking the nontrivial solution $t_{0}, t_{1}, \ldots, t_{N}$ of system (3.31) we construct the polynomial

$$
T(\lambda)=\sum_{k=0}^{N} t_{k} \lambda^{k}
$$

of degree $N$. Then substituting $s_{k+m}=\left\langle\Omega, \lambda^{k+m}\right\rangle$ in (3.31) gives

$$
\left\langle\Omega, \lambda^{m} T(\lambda)\right\rangle=0, \quad m=0,1, \ldots, N .
$$

Hence (3.30) holds for all polynomials $G(\lambda)$ with $\operatorname{deg} G(\lambda) \leq N$.

Note that the determinant of system (3.8) coincides with $D_{n-1}$. Denote by $D_{m}^{(k)}(k=$ $0,1, \ldots, m)$ the determinant that is obtained from the determinant $D_{m}$ by replacing in $D_{m}$ the $(k+1)$ th column by the column with the components $s_{m+1}, s_{m+2}, \ldots, s_{2 m+1}$. Then, solving system (3.8) by making use of the Cramer's rule, we find

$$
\chi_{n k}=-\frac{D_{n-1}^{(k)}}{D_{n-1}}, \quad k=0,1, \ldots, n-1
$$

Next, substituting the expression (3.33) of $\chi_{n k}$ into the left-hand side of (3.10), we get

$$
\alpha_{n}^{-2}=D_{n} D_{n-1}^{-1}
$$

Now if we set $D_{m}^{(m)}=\Delta_{m}$, then we get from (3.16), (3.17), by virtue of (3.33), (3.34),

$$
\begin{aligned}
& a_{n}= \pm\left(D_{n-1} D_{n+1}\right)^{1 / 2} D_{n}^{-1}, \quad n \in\{0,1, \ldots, N-2\}, \quad D_{-1}=1, \\
& b_{n}=\Delta_{n} D_{n}^{-1}-\Delta_{n-1} D_{n-1}^{-1}, \quad n \in\{0,1, \ldots, N-1\}, \quad \Delta_{-1}=0, \quad \Delta_{0}=s_{1} .
\end{aligned}
$$

Thus, if the conditions of Theorem 3 or, equivalently, the conditions of Theorem 2 are satisfied, then the entries $a_{n}, b_{n}$ of the matrix $J$ for which $\Omega$ is the generalized spectral function, are recovered by the formulas (3.35), (3.36), where $D_{n}$ is defined by (3.24) and (3.23), and $\Delta_{n}$ is the determinant obtained from the determinant $D_{n}$ by replacing in $D_{n}$ the last column by the column with the components $s_{n+1}, s_{n+2}, \ldots, s_{2 n+1}$. 


\section{Examples}

In this section we consider some simple examples to illustrate the solving of the inverse problem given above in Section 3.

Example 1. The functional

$$
\langle\Omega, G(\lambda)\rangle=\int_{0}^{1} G(\lambda) d \lambda
$$

satisfies the conditions $(i)$ and $(i i)$ of Theorem 2, but it does not satisfy the condition (iii) of this theorem.

In fact, obviously, $\langle\Omega, 1\rangle=1$. Next, let for a polynomial

$$
G(\lambda)=\sum_{k=0}^{N-1} g_{k} \lambda^{k}
$$

we have

$$
\langle\Omega, G(\lambda) H(\lambda)\rangle=\int_{0}^{1} G(\lambda) H(\lambda) d \lambda=0
$$

for all polynomials

$$
H(\lambda)=\sum_{k=0}^{N-1} h_{k} \lambda^{k}, \quad \operatorname{deg} H(\lambda)=\operatorname{deg} G(\lambda) .
$$

Taking, in particular,

$$
H(\lambda)=\sum_{k=0}^{N-1} \bar{g}_{k} \lambda^{k}
$$

where the bar over a complex number denotes the complex conjugation, we get

$$
\int_{0}^{1}|G(\lambda)|^{2} d \lambda=0
$$

and hence $G(\lambda) \equiv 0$.

The same reasoning shows that there is no nonidentically zero polynomial $T(\lambda)$ such that $\langle\Omega, G(\lambda) T(\lambda)\rangle=0$ for all polynomials $G(\lambda)$ with $\operatorname{deg} G(\lambda) \leq \operatorname{deg} H(\lambda)$.

Example 2. The functional

$$
\langle\Omega, G(\lambda)\rangle=\sum_{k=1}^{N} c_{k} G\left(\lambda_{k}\right),
$$

where $\lambda_{1}, \ldots, \lambda_{N}$ are distinct real numbers, $c_{1}, \ldots, c_{N}$ are complex numbers such that

$$
\sum_{k=1}^{N} c_{k}=1 \quad \text { and } \quad \operatorname{Re} c_{k}>0 \quad(k=1, \ldots, N),
$$

satisfies the conditions of Theorem 2 . 
In fact, obviously, $\langle\Omega, 1\rangle=1$. Next, assume that for a polynomial $G(\lambda)$ of the form (4.1) we have

$$
\langle\Omega, G(\lambda) H(\lambda)\rangle=\sum_{k=1}^{N} c_{k} G\left(\lambda_{k}\right) H\left(\lambda_{k}\right)=0
$$

for all polynomials $H(\lambda)$ of the form (4.2). If we take, in particular, $H(\lambda)$ of the form (4.3), then we get

$$
\sum_{k=1}^{N} c_{k}\left|G\left(\lambda_{k}\right)\right|^{2}=0 .
$$

Hence, taking the real part and using the condition $\operatorname{Re} c_{k}>0(k=1, \ldots, N)$, we get $G\left(\lambda_{k}\right)=0$, $k=1, \ldots, N$. Therefore $G(\lambda) \equiv 0$ because $\lambda_{1}, \ldots, \lambda_{N}$ are distinct and $G(\lambda)$ is a polynomial with $\operatorname{deg} G(\lambda) \leq N-1$.

Further, for the polynomial

$$
T(\lambda)=\left(\lambda-\lambda_{1}\right) \cdots\left(\lambda-\lambda_{N}\right)
$$

we have $\langle\Omega, G(\lambda) T(\lambda)\rangle=0$ for all polynomials $G(\lambda)$ so that the condition (iii) of Theorem 2 is also satisfied. Thus the functional $\Omega$ satisfies all the conditions of Theorem 2 .

Consider the case $N=2$ and take the functional $\Omega$ defined by the formula

$$
\langle\Omega, G(\lambda)\rangle=c G(0)+(1-c) G(1),
$$

where $c$ is any complex number such that $c \neq 0$ and $c \neq 1$. Let us solve the inverse problem for this functional by using formulas (3.35) and (3.36). We have

$$
\begin{aligned}
& s_{0}=\langle\Omega, 1\rangle=1, \quad s_{l}=\left\langle\Omega, \lambda^{l}\right\rangle=1-c \quad \text { for all } \quad l=1,2, \ldots, \\
& D_{-1}=1, \quad D_{0}=s_{0}=1 \text {, } \\
& D_{1}=\left|\begin{array}{ll}
s_{0} & s_{1} \\
s_{1} & s_{2}
\end{array}\right|=\left|\begin{array}{cc}
1 & 1-c \\
1-c & 1-c
\end{array}\right|=c(1-c), \\
& D_{2}=\left|\begin{array}{lll}
s_{0} & s_{1} & s_{2} \\
s_{1} & s_{2} & s_{3} \\
s_{2} & s_{3} & s_{4}
\end{array}\right|=\left|\begin{array}{ccc}
1 & 1-c & 1-c \\
1-c & 1-c & 1-c \\
1-c & 1-c & 1-c
\end{array}\right|=0, \\
& \Delta_{-1}=0, \quad \Delta_{0}=s_{1}=1-c, \\
& \Delta_{1}=D_{1}^{(1)}=\left|\begin{array}{ll}
s_{0} & s_{2} \\
s_{1} & s_{3}
\end{array}\right|=\left|\begin{array}{cc}
1 & 1-c \\
1-c & 1-c
\end{array}\right|=c(1-c) \text {. }
\end{aligned}
$$

Therefore the functional $\Omega$ satisfies all the conditions of Theorem 3. According to formulas (3.35) and (3.36), we find

$$
\begin{aligned}
& a_{0}= \pm\left(D_{-1} D_{1}\right)^{1 / 2} D_{0}^{-1}= \pm \sqrt{D_{1}}= \pm \sqrt{c(1-c)}, \\
& b_{0}=\Delta_{0} D_{0}^{-1}-\Delta_{-1} D_{-1}^{-1}=1-c, \\
& b_{1}=\Delta_{1} D_{1}^{-1}-\Delta_{0} D_{0}^{-1}=1-(1-c)=c .
\end{aligned}
$$

Therefore there are two matrices $J_{ \pm}$for which $\Omega$ is the spectral function:

$$
J_{ \pm}=\left[\begin{array}{ll}
b_{0} & a_{0} \\
a_{0} & b_{1}
\end{array}\right]=\left[\begin{array}{cc}
1-c & \pm \sqrt{c(1-c)} \\
\pm \sqrt{c(1-c)} & c
\end{array}\right] .
$$

The characteristic polynomials of the matrices $J_{ \pm}$have the form

$$
\operatorname{det}\left(J_{ \pm}-\lambda I\right)=\lambda(\lambda-1) .
$$


Example 3. Let $N=2$. Consider the functional $\Omega$ defined by the formula

$$
\langle\Omega, G(\lambda)\rangle=G\left(\lambda_{0}\right)+c G^{\prime}\left(\lambda_{0}\right)
$$

where $\lambda_{0}$ and $c$ are arbitrary complex numbers such that $c \neq 0$. This functional satisfies all the conditions of Theorem 2. As the polynomial $T(\lambda)$ presented in the condition (iii) of Theorem 2, we can take $T(\lambda)=\left(\lambda-\lambda_{0}\right)^{2}$.

We have

$$
\begin{aligned}
& s_{0}=\langle\Omega, 1\rangle=1, \quad s_{l}=\left\langle\Omega, \lambda^{l}\right\rangle=\lambda_{0}^{l}+c l \lambda_{0}^{l-1} \text { for } l=1,2, \ldots, \\
& D_{-1}=1, \quad D_{0}=s_{0}=1, \\
& D_{1}=\left|\begin{array}{ll}
s_{0} & s_{1} \\
s_{1} & s_{2}
\end{array}\right|=\left|\begin{array}{cc}
1 & \lambda_{0}+c \\
\lambda_{0}+c & \lambda_{0}^{2}+2 c \lambda_{0}
\end{array}\right|=-c^{2}, \\
& D_{2}=\left|\begin{array}{lll}
s_{0} & s_{1} & s_{2} \\
s_{1} & s_{2} & s_{3} \\
s_{2} & s_{3} & s_{4}
\end{array}\right|=\left|\begin{array}{ccc}
1 & \lambda_{0}+c & \lambda_{0}^{2}+2 c \lambda_{0} \\
\lambda_{0}+c & \lambda_{0}^{2}+2 c \lambda_{0} & \lambda_{0}^{3}+3 c \lambda_{0}^{2} \\
\lambda_{0}^{2}+2 c \lambda_{0} & \lambda_{0}^{3}+3 c \lambda_{0}^{2} & \lambda_{0}^{4}+4 c \lambda_{0}^{3}
\end{array}\right|=0, \\
& \Delta_{-1}=0, \quad \Delta_{0}=s_{1}=\lambda_{0}+c, \\
& \Delta_{1}=D_{1}^{(1)}=\left|\begin{array}{cc}
s_{0} & s_{2} \\
s_{1} & s_{3}
\end{array}\right|=\left|\begin{array}{cc}
1 & \lambda_{0}^{2}+2 c \lambda_{0} \\
\lambda_{0}+c & \lambda_{0}^{3}+3 c \lambda_{0}^{2}
\end{array}\right|=-2 c^{2} \lambda_{0} .
\end{aligned}
$$

Therefore the functional $\Omega$ satisfies all the conditions of Theorem 3. According to formulas (3.35) and (3.36), we find

$$
\begin{aligned}
& a_{0}= \pm\left(D_{-1} D_{1}\right)^{1 / 2} D_{0}^{-1}= \pm \sqrt{D_{1}}= \pm \sqrt{-c^{2}}= \pm i c \\
& b_{0}=\Delta_{0} D_{0}^{-1}-\Delta_{-1} D_{-1}^{-1}=\lambda_{0}+c \\
& b_{1}=\Delta_{1} D_{1}^{-1}-\Delta_{0} D_{0}^{-1}=\frac{-2 c^{2} \lambda_{0}}{-c^{2}}-\left(\lambda_{0}+c\right)=\lambda_{0}-c .
\end{aligned}
$$

Therefore the two matrices $J_{ \pm}$for which $\Omega$ is the spectral function have the form

$$
J_{ \pm}=\left[\begin{array}{cc}
b_{0} & a_{0} \\
a_{0} & b_{1}
\end{array}\right]=\left[\begin{array}{cc}
\lambda_{0}+c & \pm i c \\
\pm i c & \lambda_{0}-c
\end{array}\right]
$$

The characteristic polynomials of the matrices $J_{ \pm}$have the form

$$
\operatorname{det}\left(J_{ \pm}-\lambda I\right)=\left(\lambda-\lambda_{0}\right)^{2} .
$$

Note that if $N=3$, then the functional

$$
\langle\Omega, G(\lambda)\rangle=G\left(\lambda_{0}\right)+c_{1} G^{\prime}\left(\lambda_{0}\right)+c_{2} G^{\prime \prime}\left(\lambda_{0}\right),
$$

where $\lambda_{0}, c_{1}, c_{2}$ are complex numbers, satisfies the conditions of Theorem 2 (or Theorem 3 ) if and only if

$$
c_{2} \neq 0, \quad 2 c_{2}-c_{1}^{2} \neq 0 .
$$

\section{Structure of the generalized spectral function and spectral data}

Let $J$ be a Jacobi matrix of the form (1.1) with the entries satisfying (1.2). Next, let $\Omega$ be the generalized spectral function of $J$, defined above in Section 2. The following theorem describes the structure of $\Omega$. 
Theorem 4. Let $\lambda_{1}, \ldots, \lambda_{p}$ be all the distinct eigenvalues of the matrix $J$ and $m_{1}, \ldots, m_{p}$ be their multiplicities, respectively, as roots of the characteristic polynomial (2.6). There exist complex numbers $\beta_{k j}\left(j=1, \ldots, m_{k}, k=1, \ldots, p\right)$ uniquely determined by the matrix $J$ such that for any polynomial $G(\lambda) \in \mathbb{C}_{2 N}[\lambda]$ the formula

$$
\langle\Omega, G(\lambda)\rangle=\sum_{k=1}^{p} \sum_{j=1}^{m_{k}} \frac{\beta_{k j}}{(j-1) !} G^{(j-1)}\left(\lambda_{k}\right),
$$

holds, where $G^{(n)}(\lambda)$ denotes the nth order derivative of $G(\lambda)$ with respect to $\lambda$.

Proof. Let $J$ be a matrix of the form (1.1), (1.2). Consider the second order linear difference equation

$$
a_{n-1} y_{n-1}+b_{n} y_{n}+a_{n} y_{n+1}=\lambda y_{n}, \quad n \in\{0,1, \ldots, N-1\}, \quad a_{-1}=a_{N-1}=1,
$$

where $\left\{y_{n}\right\}_{n=-1}^{N}$ is a desired solution. Denote by $\left\{P_{n}(\lambda)\right\}_{n=-1}^{N}$ and $\left\{Q_{n}(\lambda)\right\}_{n=-1}^{N}$ the solutions of equation (5.2) satisfying the initial conditions

$$
\begin{array}{ll}
P_{-1}(\lambda)=0, & P_{0}(\lambda)=1 ; \\
Q_{-1}(\lambda)=-1, & Q_{0}(\lambda)=0 .
\end{array}
$$

For each $n \geq 0, P_{n}(\lambda)$ is a polynomial of degree $n$ and is called a polynomial of first kind (note that $P_{n}(\lambda)$ is the same polynomial as in Section 2) and $Q_{n}(\lambda)$ is a polynomial of degree $n-1$ and is known as a polynomial of second kind.

Let us set

$$
M(\lambda)=-\frac{Q_{N}(\lambda)}{P_{N}(\lambda)}
$$

Then it is straightforward to verify that the entries $R_{n m}(\lambda)$ of the matrix $R(\lambda)=(J-\lambda I)^{-1}$ (resolvent of $J$ ) are of the form

$$
R_{n m}(\lambda)= \begin{cases}P_{n}(\lambda)\left[Q_{m}(\lambda)+M(\lambda) P_{m}(\lambda)\right], & 0 \leq n \leq m \leq N-1, \\ P_{m}(\lambda)\left[Q_{n}(\lambda)+M(\lambda) P_{n}(\lambda)\right], & 0 \leq m \leq n \leq N-1 .\end{cases}
$$

Let $f$ be an arbitrary element (column vector) of $\mathbb{C}^{N}$, with the components $f_{0}, f_{1}, \ldots, f_{N-1}$. Since

$$
R(\lambda) f=-\frac{f}{\lambda}+O\left(\frac{1}{\lambda^{2}}\right)
$$

as $|\lambda| \rightarrow \infty$, we have for each $n \in\{0,1, \ldots, N-1\}$,

$$
f_{n}=-\frac{1}{2 \pi i} \int_{\Gamma_{r}}\left\{\sum_{m=0}^{N-1} R_{n m}(\lambda) f_{m}\right\} d \lambda+\int_{\Gamma_{r}} O\left(\frac{1}{\lambda^{2}}\right) d \lambda,
$$

where $r$ is a sufficiently large positive number, $\Gamma_{r}$ is the circle in the $\lambda$-plane of radius $r$ centered at the origin.

Denote by $\lambda_{1}, \ldots, \lambda_{p}$ all the distinct roots of the polynomial $P_{N}(\lambda)$ (which coincides by (2.6) with the characteristic polynomial of the matrix $J$ up to a constant factor) and by $m_{1}, \ldots, m_{p}$ their multiplicities, respectively:

$$
P_{N}(\lambda)=c\left(\lambda-\lambda_{1}\right)^{m_{1}} \cdots\left(\lambda-\lambda_{p}\right)^{m_{p}},
$$


where $c$ is a constant. We have $1 \leq p \leq N$ and $m_{1}+\cdots+m_{p}=N$. By (5.8), we can rewrite the rational function $Q_{N}(\lambda) / P_{N}(\lambda)$ as the sum of partial fractions:

$$
\frac{Q_{N}(\lambda)}{P_{N}(\lambda)}=\sum_{k=1}^{p} \sum_{j=1}^{m_{k}} \frac{\beta_{k j}}{\left(\lambda-\lambda_{k}\right)^{j}}
$$

where $\beta_{k j}$ are some uniquely determined complex numbers depending on the matrix $J$. Substituting (5.6) in (5.7) and taking into account (5.5), (5.9) we get, applying the residue theorem and passing then to the limit as $r \rightarrow \infty$,

$$
f_{n}=\sum_{k=1}^{p} \sum_{j=1}^{m_{k}} \frac{\beta_{k j}}{(j-1) !}\left\{\frac{d^{j-1}}{d \lambda^{j-1}}\left[F(\lambda) P_{n}(\lambda)\right]\right\}_{\lambda=\lambda_{k}}, \quad n \in\{0,1, \ldots, N-1\},
$$

where

$$
F(\lambda)=\sum_{m=0}^{N-1} f_{m} P_{m}(\lambda)
$$

Now define on $\mathbb{C}_{2 N}[\lambda]$ the functional $\Omega$ by the formula

$$
\langle\Omega, G(\lambda)\rangle=\sum_{k=1}^{p} \sum_{j=1}^{m_{k}} \frac{\beta_{k j}}{(j-1) !} G^{(j-1)}\left(\lambda_{k}\right), \quad G(\lambda) \in \mathbb{C}_{2 N}[\lambda] .
$$

Then formula (5.10) can be written in the form

$$
f_{n}=\left\langle\Omega, F(\lambda) P_{n}(\lambda)\right\rangle, \quad n \in\{0,1, \ldots, N-1\} .
$$

From here by (5.11) and the arbitrariness of $\left\{f_{m}\right\}_{m=0}^{N-1}$ it follows that the "orthogonality" relation

$$
\left\langle\Omega, P_{m}(\lambda) P_{n}(\lambda)\right\rangle=\delta_{m n}, \quad m, n \in\{0,1, \ldots, N-1\},
$$

holds. Further, in virtue of (5.8) and (5.12) we have also

$$
\left\langle\Omega, P_{m}(\lambda) P_{N}(\lambda)\right\rangle=0, \quad m \in\{0,1, \ldots, N\} .
$$

These mean by Theorem 1 that the generalized spectral function of the matrix $J$ has the form (5.12).

Definition 2. The collection of the quantities

$$
\left\{\lambda_{k}, \beta_{k j}\left(j=1, \ldots, m_{k}, k=1, \ldots, p\right)\right\},
$$

determining the structure of the generalized spectral function of the matrix $J$ according to Theorem 4, we call the spectral data of the matrix $J$. For each $k \in\{1, \ldots, p\}$ the sequence

$$
\left\{\beta_{k 1}, \ldots, \beta_{k m_{k}}\right\}
$$

we call the normalizing chain (of the matrix $J$ ) associated with the eigenvalue $\lambda_{k}$ (the sense of "normalizing" will be clear below in Section 8). 
If we delete the first row and the first column of the matrix $J$ given in (1.1), then we get the new matrix

$$
J^{(1)}=\left[\begin{array}{ccccccc}
b_{0}^{(1)} & a_{0}^{(1)} & 0 & \ldots & 0 & 0 & 0 \\
a_{0}^{(1)} & b_{1}^{(1)} & a_{1}^{(1)} & \ldots & 0 & 0 & 0 \\
0 & a_{1}^{(1)} & b_{2}^{(1)} & \ldots & 0 & 0 & 0 \\
\vdots & \vdots & \vdots & \ddots & \vdots & \vdots & \vdots \\
0 & 0 & 0 & \ldots & b_{N-4}^{(1)} & a_{N-4}^{(1)} & 0 \\
0 & 0 & 0 & \ldots & a_{N-4}^{(1)} & b_{N-3}^{(1)} & a_{N-3}^{(1)} \\
0 & 0 & 0 & \ldots & 0 & a_{N-3}^{(1)} & b_{N-2}^{(1)}
\end{array}\right],
$$

where

$$
\begin{array}{ll}
a_{n}^{(1)}=a_{n+1}, & n \in\{0,1, \ldots, N-3\}, \\
b_{n}^{(1)}=b_{n+1}, & n \in\{0,1, \ldots, N-2\} .
\end{array}
$$

The matrix $J^{(1)}$ is called the first truncated matrix (with respect to the matrix $J$ ).

Theorem 5. The normalizing numbers $\beta_{k j}$ of the matrix $J$ can be calculated by decomposing the rational function

$$
-\frac{\operatorname{det}\left(J^{(1)}-\lambda I\right)}{\operatorname{det}(J-\lambda I)}
$$

into partial fractions.

Proof. Let us denote the polynomials of the first and the second kinds, corresponding to the matrix $J^{(1)}$, by $P_{n}^{(1)}(\lambda)$ and $Q_{n}^{(1)}(\lambda)$, respectively. It is easily seen that

$$
\begin{aligned}
& P_{n}^{(1)}(\lambda)=a_{0} Q_{n+1}(\lambda), \quad n \in\{0,1, \ldots, N-1\}, \\
& Q_{n}^{(1)}(\lambda)=\frac{1}{a_{0}}\left\{\left(\lambda-b_{0}\right) Q_{n+1}(\lambda)-P_{n+1}(\lambda)\right\}, \quad n \in\{0,1, \ldots, N-1\} .
\end{aligned}
$$

Indeed, both sides of each of these equalities are solutions of the same difference equation

$$
a_{n-1}^{(1)} y_{n-1}+b_{n}^{(1)} y_{n}+a_{n}^{(1)} y_{n+1}=\lambda y_{n}, \quad n \in\{0,1, \ldots, N-2\}, \quad a_{N-2}^{(1)}=1,
$$

and the sides coincide for $n=-1$ and $n=0$. Therefore the equality holds by the uniqueness theorem for solutions.

Consequently, taking into account Lemma 1 and using (5.16), we have

$$
\operatorname{det}\left(J^{(1)}-\lambda I\right)=(-!)^{N-1} a_{0}^{(1)} a_{1}^{(1)} \cdots a_{N-3}^{(1)} P_{N-1}^{(1)}(\lambda)=(-1)^{N-1} a_{1} \cdots a_{N-2} a_{0} Q_{N}(\lambda) .
$$

Comparing this with (2.6), we get

$$
\frac{Q_{N}(\lambda)}{P_{N}(\lambda)}=-\frac{\operatorname{det}\left(J^{(1)}-\lambda I\right)}{\operatorname{det}(J-\lambda I)}
$$

so that the statement of the theorem follows from (5.9). 


\section{Inverse problem from the spectral data}

By the inverse spectral problem is meant the problem of recovering matrix $J$, i.e. its entries $a_{n}$ and $b_{n}$, from the spectral data.

Theorem 6. Let an arbitrary collection of complex numbers

$$
\left\{\lambda_{k}, \beta_{k j}\left(j=1, \ldots, m_{k}, k=1, \ldots, p\right)\right\}
$$

be given, where $\lambda_{1}, \lambda_{2}, \ldots, \lambda_{p}(1 \leq p \leq N)$ are distinct, $1 \leq m_{k} \leq N$, and $m_{1}+\cdots+m_{p}=N$. In order for this collection to be the spectral data for some Jacobi matrix $J$ of the form (1.1) with entries belonging to the class (1.2), it is necessary and sufficient that the following two conditions be satisfied:

(i) $\sum_{k=1}^{p} \beta_{k 1}=1$;

(ii) $D_{n} \neq 0$, for $n \in\{1,2, \ldots, N-1\}$, and $D_{N}=0$, where $D_{n}$ is defined by (3.24) in which

$$
\begin{gathered}
s_{l}=\sum_{k=1}^{p} \sum_{j=1}^{n_{k l}}\left(\begin{array}{c}
l \\
j-1
\end{array}\right) \beta_{k j} \lambda_{k}^{l-j+1}, \\
n_{k l}=\min \left\{m_{k}, l+1\right\},\left(\begin{array}{c}
l \\
j-1
\end{array}\right) \text { is a binomial coefficient. }
\end{gathered}
$$

Proof. The necessity of conditions of the theorem follows from Theorem 3 because the generalized spectral function of the matrix $J$ is defined by the spectral data according to formula (5.1) and therefore the quantity (6.2) coincides with $\left\langle\Omega, \lambda^{l}\right\rangle$. Besides,

$$
\sum_{k=1}^{p} \beta_{k 1}=\langle\Omega, 1\rangle=s_{0}=D_{0} .
$$

Note that the condition (iii) of Theorem 2 holds with

$$
T(\lambda)=\left(\lambda-\lambda_{1}\right)^{m_{1}} \cdots\left(\lambda-\lambda_{p}\right)^{m_{p}} .
$$

Let us prove the sufficiency. Assume that we have a collection of quantities (6.1) satisfying the conditions of the theorem. Using these data we construct the functional $\Omega$ on $\mathbb{C}_{2 N}[\lambda]$ by formula (5.1). Then this functional $\Omega$ satisfies the conditions of Theorem 3 and therefore there exists a matrix $J$ of the form (1.1), (1.2) for which $\Omega$ is the generalized spectral function. Now we have to prove that the collection $(6.1)$ is the spectral data for the recovered matrix $J$. For this purpose we define the polynomials $P_{-1}(\lambda), P_{0}(\lambda), \ldots, P_{N}(\lambda)$ as the solution of equation (5.2), constructed by means of the matrix $J$, under the initial conditions (5.3). Then the relations (2.7), (2.8) and the equalities

$$
\begin{aligned}
& a_{n}=\left\langle\Omega, \lambda P_{n}(\lambda) P_{n+1}(\lambda)\right\rangle, \quad n \in\{0,1, \ldots, N-2\}, \\
& b_{n}=\left\langle\Omega, \lambda P_{n}^{2}(\lambda)\right\rangle, \quad n \in\{0,1, \ldots, N-1\}
\end{aligned}
$$

hold. We show that (5.8) holds which will mean, in particular, that $\lambda_{1}, \ldots, \lambda_{p}$ are eigenvalues of the matrix $J$ with the multiplicities $m_{1}, \ldots, m_{p}$, respectively.

Let $T(\lambda)$ be defined by (6.3). Let us show that there exists a constant $c$ such that

$$
a_{N-2} P_{N-2}(\lambda)+b_{N-1} P_{N-1}(\lambda)+c T(\lambda)=\lambda P_{N-1}(\lambda)
$$

for all $\lambda \in \mathbb{C}$. If we prove this, then from here and (5.2) with $y_{k}=P_{k}(\lambda)$ and $n=N-1$ we get that $P_{N}(\lambda)=c T(\lambda)$. 
Since $\operatorname{deg} P_{n}(\lambda)=n(0 \leq n \leq N-1), \operatorname{deg} T(\lambda)=m_{1}+\cdots+m_{p}=N$, the polynomials $P_{0}(\lambda), \ldots, P_{N-1}(\lambda), T(\lambda)$ form a basis of the linear space of all polynomials of degree $\leq N$. Therefore we have the decomposition

$$
\lambda P_{N-1}(\lambda)=c T(\lambda)+\sum_{n=0}^{N-1} c_{n} P_{n}(\lambda)
$$

where $c, c_{0}, c_{1}, \ldots, c_{N-1}$ are some constants. By (6.3) and (5.1) it follows that

$$
\left\langle\Omega, T(\lambda) P_{n}(\lambda)\right\rangle=0, \quad n \in\{0,1, \ldots, N\} .
$$

Hence taking into account the relations (2.7), (2.8) and (6.4), (6.5), we find from (6.7) that

$$
c_{n}=0 \quad(0 \leq n \leq N-3), \quad c_{N-2}=a_{N-2}, \quad c_{N-1}=b_{N-1} .
$$

So (6.6) is shown.

It remains to show that for each $k \in\{1, \ldots, p\}$ the sequence $\left\{\beta_{k 1}, \ldots, \beta_{k m_{k}}\right\}$ is the normalizing chain of the matrix $J$ associated with the eigenvalue $\lambda_{k}$. Since we have already shown that $\lambda_{k}$ is an eigenvalue of the matrix $J$ of the multiplicity $m_{k}$, the normalizing chain of $J$ associated with the eigenvalue $\lambda_{k}$ has the form $\left\{\widetilde{\beta}_{k 1}, \ldots, \widetilde{\beta}_{k m_{k}}\right\}$. Therefore for $\langle\Omega, G(\lambda)\rangle$ we have an equality of the form (5.1) in which $\beta_{k j}$ is replaced by $\widetilde{\beta}_{k j}$. Subtracting these two equalities for $\langle\Omega, G(\lambda)\rangle$ each from other we get that

$$
\sum_{k=1}^{p} \sum_{j=1}^{m_{k}} \frac{\beta_{k j}-\widetilde{\beta}_{k j}}{(j-1) !} G^{(j-1)}\left(\lambda_{k}\right)=0 \quad \text { for all } \quad G(\lambda) \in \mathbb{C}_{2 N}[\lambda] .
$$

Since the values $G^{(j-1)}\left(\lambda_{k}\right)$ can be arbitrary numbers, we get that $\beta_{k j}=\widetilde{\beta}_{k j}$ for all $k$ and $j$.

Under the conditions of Theorem 6 the entries $a_{n}$ and $b_{n}$ of the matrix $J$ for which the collection (6.1) is spectral data, are recovered by formulas (3.35), (3.36).

\section{Characterization of generalized spectral functions of real Jacobi matrices}

In this section, we characterize generalized spectral functions of real Jacobi matrices among the generalized spectral functions of complex Jacobi matrices. Let $m$ be a nonnegative integer. Denote by $\mathbb{R}_{2 m}[\lambda]$ the ring of all polynomials in $\lambda$ of degree $\leq 2 m$ with real coefficients.

Definition 3. A linear functional $\Omega$ defined on the space $\mathbb{C}_{2 m}[\lambda]$ is said to be positive if

$$
\langle\Omega, G(\lambda)\rangle>0
$$

for all polynomials $G(\lambda) \in \mathbb{R}_{2 m}[\lambda]$, which are not identically zero and which satisfy the inequality

$$
G(\lambda) \geq 0, \quad-\infty<\lambda<\infty
$$

Lemma 2. If $\Omega$ is a positive functional on $\mathbb{C}_{2 m}[\lambda]$, then it takes only real values on $\mathbb{R}_{2 m}[\lambda]$.

Proof. Since the functional $\Omega$ is positive, the values $\left\langle\Omega, \lambda^{2 k}\right\rangle, k \in\{0,1, \ldots, m\}$ are real (moreover they are positive). Next, the monomial $\lambda^{2 k-1}, k \in\{1,2, \ldots, m\}$ is represented as a difference of two nonnegative polynomials of degree $2 k$ :

$$
2 \lambda^{2 k-1}=\lambda^{2 k-2}(\lambda+1)^{2}-\lambda^{2 k-2}\left(\lambda^{2}+1\right) .
$$

Therefore the values $\left\langle\Omega, \lambda^{2 k-1}\right\rangle, k \in\{1,2, \ldots, m\}$ are also real to be a difference of two positive numbers. Thus, $\left\langle\Omega, \lambda^{n}\right\rangle$ is real for any $n \in\{0,1, \ldots, 2 m\}$. Hence $\langle\Omega, G(\lambda)\rangle$ is real for any $G(\lambda) \in \mathbb{R}_{2 m}[\lambda]$ 
Lemma 3. A linear functional $\Omega$ on $\mathbb{C}_{2 m}[\lambda]$ is positive if and only if $D_{n}>0$ for all $n \in$ $\{0,1, \ldots, m\}$, where

$$
D_{n}=\left|\begin{array}{cccc}
s_{0} & s_{1} & \cdots & s_{n} \\
s_{1} & s_{2} & \cdots & s_{n+1} \\
\vdots & \vdots & \ddots & \vdots \\
s_{n} & s_{n+1} & \cdots & s_{2 n}
\end{array}\right|, \quad n=0,1, \ldots, m
$$

in which

$$
s_{l}=\left\langle\Omega, \lambda^{l}\right\rangle, \quad l=0,1, \ldots, 2 m .
$$

Proof. Any polynomial $G(\lambda) \in \mathbb{R}_{2 m}[\lambda]$ which is not identically zero and which satisfies the inequality

$$
G(\lambda) \geq 0, \quad-\infty<\lambda<\infty
$$

can be represented in the form

$$
G(\lambda)=[A(\lambda)]^{2}+[B(\lambda)]^{2},
$$

where $A(\lambda), B(\lambda)$ are polynomials of degrees $\leq m$ with real coefficients. Indeed, it follows from (7.1) that the polynomial $G(\lambda)$ has even degree: $\operatorname{deg} G(\lambda)=2 p$, where $p \leq m$. Therefore its decomposition into linear factors has the form

$$
G(\lambda)=c \prod_{k=1}^{p}\left(\lambda-\alpha_{k}-i \beta_{k}\right)\left(\lambda-\alpha_{k}+i \beta_{k}\right),
$$

where $c>0, \beta_{k} \geq 0, \alpha_{k}$ are real (among the roots $\alpha_{k}+i \beta_{k}$, of course, may be equal). Now setting

$$
\sqrt{c} \prod_{k=1}^{p}\left(\lambda-\alpha_{k}-i \beta_{k}\right)=A(\lambda)+i B(\lambda),
$$

we get that the polynomials $A(\lambda), B(\lambda)$ have real coefficients and (7.2) holds.

Now writing

$$
A(\lambda)=\sum_{k=1}^{p} x_{k} \lambda^{k}, \quad B(\lambda)=\sum_{k=1}^{p} y_{k} \lambda^{k},
$$

where $x_{k}, y_{k}$ are real numbers, we find

$$
\langle\Omega, G(\lambda)\rangle=\sum_{j, k=0}^{p} s_{j+k} x_{j} x_{k}+\sum_{j, k=0}^{p} s_{j+k} y_{j} y_{k} .
$$

This implies the statement of the lemma.

Theorem 7. In order for a given linear functional $\Omega$ on $\mathbb{C}_{2 N}[\lambda]$, to be the generalized spectral function for a real Jacobi matrix of the form (1.1), (1.3) it is necessary and sufficient that the following three conditions be satisfied:

(i) $\langle\Omega, 1\rangle=1$;

(ii) $\Omega$ is positive on $\mathbb{C}_{2 N-2}[\lambda]$; 
(iii) there exists a polynomial $T(\lambda)$ of degree $N$ such that $\langle\Omega, G(\lambda) T(\lambda)\rangle=0$ for all polynomials $G(\lambda)$ with $\operatorname{deg} G(\lambda) \leq N$.

Proof. Necessity. The condition $\langle\Omega, 1\rangle=1$ follows from (2.7) with $m=n=0$. To prove positivity on $\mathbb{C}_{2 N-2}[\lambda]$ of the generalized spectral function $\Omega$ of the real Jacobi matrix $J$, take an arbitrary polynomial $G(\lambda) \in \mathbb{R}_{2 N-2}[\lambda]$ which is not identically zero and which satisfies the inequality

$$
G(\lambda) \geq 0, \quad-\infty<\lambda<\infty
$$

This polynomial can be represented in the form (see the proof of Lemma 3)

$$
G(\lambda)=[A(\lambda)]^{2}+[B(\lambda)]^{2},
$$

where $A(\lambda), B(\lambda)$ are polynomials of degrees $\leq N-1$ with real coefficients. Since the polynomials $P_{0}(\lambda), P_{1}(\lambda), \ldots, P_{N-1}(\lambda)$ have real coefficients (because $J$ is a real matrix) and they form a basis of $\mathbb{R}_{N-1}[\lambda]$, we can write the decompositions

$$
A(\lambda)=\sum_{k=1}^{N-1} c_{k} P_{k}(\lambda), \quad B(\lambda)=\sum_{k=1}^{N-1} d_{k} P_{k}(\lambda)
$$

where $c_{k}, d_{k}$ are real numbers not all zero. Therefore using the "orthogonality" property (2.7) we get from (7.3),

$$
\langle\Omega, G(\lambda)\rangle=\sum_{j, k=0}^{N-1}\left(c_{k}^{2}+d_{k}^{2}\right)>0 .
$$

The property of $\Omega$ indicated in the condition (iii) of the theorem follows from (2.8) if we take $T(\lambda)=P_{N}(\lambda)$.

Sufficiency. It follows from the conditions of the theorem that all the conditions of Theorem 2 are satisfied. In fact, we need to verify only the condition $(i i)$ of Theorem 2 . Let for some polynomial $G(\lambda), \operatorname{deg} G(\lambda)=n \leq N-1$,

$$
\langle\Omega, G(\lambda) H(\lambda)\rangle=0
$$

for all polynomials $H(\lambda)$, deg $H(\lambda)=n$. We have to show that then $G(\lambda) \equiv 0$. Setting

$$
G(\lambda)=\sum_{k=0}^{n} g_{k} \lambda^{k}, \quad H(\lambda)=\sum_{j=0}^{n} h_{j} \lambda^{j},
$$

we get from (7.4) that

$$
\sum_{j=0}^{n} h_{j}\left(\sum_{k=0}^{n} g_{k} s_{k+j}\right)=0 .
$$

Since $h_{0}, h_{1}, \ldots, h_{n}\left(h_{n} \neq 0\right)$ are arbitrary, the last equation gives

$$
\sum_{k=0}^{n} g_{k} s_{k+j}=0, \quad j=0,1, \ldots, n .
$$

This is a linear homogeneous system of algebraic equations with respect to $g_{0}, g_{1}, \ldots, g_{n}$ and the determinant of this system coincides with the determinant $D_{n}$. From the condition $(i i)$ of the 
theorem it follows by Lemma 3 that $D_{n}>0$. So $D_{n} \neq 0$ and hence system (7.5) has only the trivial solution $g_{0}=g_{1}=\cdots=g_{n}$.

Thus, all the conditions of Theorem 2 are satisfied. Therefore there exists, generally speaking, a complex Jacobi matrix $J$ of the form (1.1), (1.2) for which $\Omega$ is the generalized spectral function. This matrix $J$ is constructed by using formulas (3.35), (3.36). It remains to show that the matrix $J$ is real. But this follows from the fact that by Lemma 2 and Lemma 3 we have $D_{n}>0$ for $n \in\{0,1, \ldots, N-1\}$ and the determinants $\Delta_{n}$ are real. Therefore formulas (3.35), (3.36) imply that the matrix $J$ is real.

If we take into account Lemma 3 , then it is easily seen from the proof of Theorem 3 that Theorem 7 is equivalent to the following theorem.

Theorem 8. In order for a given linear functional $\Omega$, defined on $\mathbb{C}_{2 N}[\lambda]$, to be the generalized spectral function for some real Jacobi matrix $J$ of the form (1.1) with entries belonging to the class (1.3), it is necessary and sufficient that

$$
D_{0}=1, \quad D_{n}>0 \quad(n=1,2, \ldots, N-1), \quad \text { and } \quad D_{N}=0 \text {, }
$$

where $D_{n}$ is defined by (3.24) and (3.23).

Under the conditions of Theorem 8 the entries $a_{n}$ and $b_{n}$ of the matrix $J$ for which the functional $\Omega$ is the spectral function are recovered by formulas (3.35), (3.36).

\section{Structure of generalized spectral functions of real Jacobi matrices}

First we prove two lemmas which hold for any complex Jacobi matrix $J$ of the form (1.1), (1.2). Having the matrix $J$ consider the difference equation (5.2) and let $\left\{P_{n}(\lambda)\right\}_{n=-1}^{N}$ and $\left\{Q_{n}(\lambda)\right\}_{n=-1}^{N}$ be the solutions of this equation satisfying the initial conditions (5.3) and (5.4), respectively.

Lemma 4. The equation

$$
P_{N-1}(\lambda) Q_{N}(\lambda)-P_{N}(\lambda) Q_{N-1}(\lambda)=1
$$

holds.

Proof. Multiply first of the equations

$$
\begin{aligned}
& a_{n-1} P_{n-1}(\lambda)+b_{n} P_{n}(\lambda)+a_{n} P_{n+1}(\lambda)=\lambda P_{n}(\lambda), \\
& n \in\{0,1, \ldots, N-1\}, \quad a_{-1}=a_{N-1}=1, \\
& a_{n-1} Q_{n-1}(\lambda)+b_{n} Q_{n}(\lambda)+a_{n} Q_{n+1}(\lambda)=\lambda Q_{n}(\lambda), \\
& n \in\{0,1, \ldots, N-1\}, \quad a_{-1}=a_{N-1}=1,
\end{aligned}
$$

by $Q_{n}(\lambda)$ and second by $P_{n}(\lambda)$ and subtract the second result from the first to get

$$
\begin{aligned}
& a_{n-1}\left[P_{n-1}(\lambda) Q_{n}(\lambda)-P_{n}(\lambda) Q_{n-1}(\lambda)\right] \\
& \quad=a_{n}\left[P_{n}(\lambda) Q_{n+1}(\lambda)-P_{n+1}(\lambda) Q_{n}(\lambda)\right], \quad n \in\{0,1, \ldots, N-1\} .
\end{aligned}
$$

This means that the expression (Wronskian of the solutions $P_{n}(\lambda)$ and $Q_{n}(\lambda)$ )

$$
a_{n}\left[P_{n}(\lambda) Q_{n+1}(\lambda)-P_{n+1}(\lambda) Q_{n}(\lambda)\right]
$$

does not depend on $n \in\{-1,0,1, \ldots, N-1\}$. On the other hand the value of this expression at $n=-1$ is equal to 1 by (5.3), (5.4), and $a_{-1}=1$. Therefore

$$
a_{n}\left[P_{n}(\lambda) Q_{n+1}(\lambda)-P_{n+1}(\lambda) Q_{n}(\lambda)\right]=1 \quad \text { for all } n \in\{-1,0,1, \ldots, N-1\} .
$$

Putting here, in particular, $n=N-1$, we arrive at (8.1). 
Lemma 5. The equation

$$
P_{N-1}(\lambda) P_{N}^{\prime}(\lambda)-P_{N}(\lambda) P_{N-1}^{\prime}(\lambda)=\sum_{n=0}^{N-1} P_{n}^{2}(\lambda)
$$

holds, where the prime denotes the derivative with respect to $\lambda$.

Proof. Differentiating equation (8.2) with respect to $\lambda$, we get

$$
\begin{aligned}
& a_{n-1} P_{n-1}^{\prime}(\lambda)+b_{n} P_{n}^{\prime}(\lambda)+a_{n} P_{n+1}^{\prime}(\lambda)=\lambda P_{n}^{\prime}(\lambda)+P_{n}(\lambda), \\
& n \in\{0,1, \ldots, N-1\}, \quad a_{-1}=a_{N-1}=1 .
\end{aligned}
$$

Multiplying equation (8.2) by $P_{n}^{\prime}(\lambda)$ and equation (8.4) by $P_{n}(\lambda)$, and subtracting the left and right members of the resulting equations, we get

$$
\begin{aligned}
& a_{n-1}\left[P_{n-1}(\lambda) P_{n}^{\prime}(\lambda)-P_{n}(\lambda) P_{n-1}^{\prime}(\lambda)\right]-a_{n}\left[P_{n}(\lambda) P_{n+1}^{\prime}(\lambda)-P_{n+1}(\lambda) P_{n}^{\prime}(\lambda)\right]=-P_{n}^{2}(\lambda), \\
& \quad n \in\{0,1, \ldots, N-1\} .
\end{aligned}
$$

Summing the last equation for the values $n=0,1, \ldots, m(m \leq N-1)$ and using the initial conditions (5.3), we obtain

$$
a_{m}\left[P_{m}(\lambda) P_{m+1}^{\prime}(\lambda)-P_{m+1}(\lambda) P_{m}^{\prime}(\lambda)\right]=\sum_{n=0}^{m} P_{n}^{2}(\lambda), \quad m \in\{0,1, \ldots, N-1\} .
$$

Setting here, in particular, $m=N-1$ and taking into account $a_{N-1}=1$, we get (8.3).

Now we consider real Jacobi matrices of the form (1.1), (1.3).

Lemma 6. For any real Jacobi matrix $J$ of the form (1.1), (1.3) the roots of the polynomial $P_{N}(\lambda)$ are simple.

Proof. Let $\lambda_{0}$ be a root of the polynomial $P_{N}(\lambda)$. The root $\lambda_{0}$ is an eigenvalue of the matrix $J$ by (2.6) and hence it is real by Hermiticity of $J$. Putting $\lambda=\lambda_{0}$ in (8.3) and using $P_{N}\left(\lambda_{0}\right)=0$, we get

$$
P_{N-1}\left(\lambda_{0}\right) P_{N}^{\prime}\left(\lambda_{0}\right)=\sum_{n=0}^{N-1} P_{n}^{2}\left(\lambda_{0}\right)
$$

The right-hand side of (8.5) is different from zero because the polynomials $P_{n}(\lambda)$ have real coefficients and hence are real for real values of $\lambda$, and $P_{0}(\lambda)=1$. Consequently $P_{N}^{\prime}\left(\lambda_{0}\right) \neq 0$, that is, the root $\lambda_{0}$ of the polynomial $P_{N}(\lambda)$ is simple.

Lemma 7. Any real Jacobi matrix $J$ of the form (1.1), (1.3) has precisely $N$ real and distinct eigenvalues.

Proof. The reality of eigenvalues of $J$ follows from its Hermiticity. Next, the eigenvalues of $J$ coincide, by (2.6), with the roots of the polynomial $P_{N}(\lambda)$. This polynomial of degree $N$ has $N$ roots. These roots are pairwise distinct by Lemma 6 .

The following theorem describes the structure of generalized spectral functions of real Jacobi matrices. 
Theorem 9. Let $J$ be a real Jacobi matrix of the form (1.1), (1.3) and $\Omega$ be its generalized spectral function. Then for any $G(\lambda) \in \mathbb{C}_{2 N}[\lambda]$

$$
\langle\Omega, G(\lambda)\rangle=\sum_{k=1}^{N} \beta_{k} G\left(\lambda_{k}\right),
$$

where $\lambda_{1}, \ldots, \lambda_{N}$ are the eigenvalues of the matrix $J$ and $\beta_{1}, \ldots, \beta_{N}$ are positive real numbers uniquely determined by the matrix $J$.

Proof. By Lemma 6, the roots $\lambda_{1}, \ldots, \lambda_{N}$ of the polynomial $P_{N}(\lambda)$ are simple. Therefore the formula (8.6) follows from (5.1) and the decomposition (5.9) takes the form

$$
\frac{Q_{N}(\lambda)}{P_{N}(\lambda)}=\sum_{k=1}^{N} \frac{\beta_{k}}{\lambda-\lambda_{k}} .
$$

Hence

$$
Q_{N}\left(\lambda_{k}\right)=\beta_{k} P_{N}^{\prime}\left(\lambda_{k}\right) .
$$

On the other hand, putting $\lambda=\lambda_{k}$ in (8.1) and (8.3) and taking into account that $P_{N}\left(\lambda_{k}\right)=0$, we get

$$
\begin{aligned}
& P_{N-1}\left(\lambda_{k}\right) Q_{N}\left(\lambda_{k}\right)=1, \\
& P_{N-1}\left(\lambda_{k}\right) P_{N}^{\prime}\left(\lambda_{k}\right)=\sum_{n=0}^{N-1} P_{n}^{2}\left(\lambda_{k}\right),
\end{aligned}
$$

respectively. Comparing (8.7), (8.8), and (8.9), we find that

$$
\beta_{k}=\left\{\sum_{n=0}^{N-1} P_{n}^{2}\left(\lambda_{k}\right)\right\}^{-1},
$$

whence we get, in particular, that $\beta_{k}>0$.

Since $\left\{P_{n}\left(\lambda_{k}\right)\right\}_{n=0}^{N-1}$ is an eigenvector of the matrix $J$ corresponding to the eigenvalue $\lambda_{k}$, it is natural, according to the formula (8.10), to call the numbers $\beta_{k}$ the normalizing numbers of the matrix $J$.

Definition 4. The collection of the eigenvalues and normalizing numbers

$$
\left\{\lambda_{k}, \beta_{k}(k=1, \ldots, N)\right\}
$$

of the matrix $J$ of the form (1.1), (1.3) we call the spectral data of this matrix.

Remark 2. Assuming that $\lambda_{1}<\lambda_{2}<\cdots<\lambda_{N}$, let us introduce the nondecreasing step function $\omega(\lambda)$ on $(-\infty, \infty)$ by

$$
\omega(\lambda)=\sum_{\lambda_{k} \leq \lambda} \beta_{k}
$$

where $\omega(\lambda)=0$ if there is no $\lambda_{k} \leq \lambda$. So the eigenvalues of the matrix $J$ are the points of increase of the function $\omega(\lambda)$. Then equality (8.6) can be written as

$$
\langle\Omega, G(\lambda)\rangle=\int_{-\infty}^{\infty} G(\lambda) d \omega(\lambda)
$$


where the integral is a Stieltjes integral. Therefore the orthogonality relation (5.14) can be written as

$$
\int_{-\infty}^{\infty} P_{m}(\lambda) P_{n}(\lambda) d \omega(\lambda)=\delta_{m n}, \quad m, n \in\{0,1, \ldots, N-1\}
$$

and the expansion formula (5.13) as

$$
f_{n}=\int_{-\infty}^{\infty} F(\lambda) P_{n}(\lambda) d \omega(\lambda), \quad n \in\{0,1, \ldots, N-1\}
$$

where $F(\lambda)$ is defined by (5.11):

$$
F(\lambda)=\sum_{m=0}^{N-1} f_{m} P_{m}(\lambda)
$$

Such function $\omega(\lambda)$ is known as a spectral function (see, e.g., [21]) of the operator (matrix) $J$. This explains the source of the term "generalized spectral function" used in the complex case.

\section{Inverse spectral problem for real Jacobi matrices}

By the inverse spectral problem for real Jacobi matrices we mean the problem of recovering the matrix, i.e. its entries, from the spectral data.

Theorem 10. Let an arbitrary collection of numbers

$$
\left\{\lambda_{k}, \beta_{k}(k=1, \ldots, N)\right\}
$$

be given. In order for this collection to be the spectral data for a real Jacobi matrix $J$ of the form (1.1) with entries belonging to the class (1.3), it is necessary and sufficient that the following two conditions be satisfied:

(i) The numbers $\lambda_{1}, \ldots, \lambda_{N}$ are real and distinct.

(ii) The numbers $\beta_{1}, \ldots, \beta_{N}$ are positive and such that $\sum_{k=1}^{N} \beta_{k}=1$.

Proof. The necessity of the conditions of the theorem was proved above. To prove the sufficiency, assume that we have a collection of quantities (9.1) satisfying the conditions of the theorem. Using these data we construct the functional $\Omega$ on $\mathbb{C}_{2 N}[\lambda]$ by the formula

$$
\langle\Omega, G(\lambda)\rangle=\sum_{k=1}^{N} \beta_{k} G\left(\lambda_{k}\right), \quad G(\lambda) \in \mathbb{C}_{2 N}[\lambda] .
$$

Then this functional $\Omega$ satisfies the conditions of Theorem 7 . Indeed, we have

$$
\langle\Omega, 1\rangle=\sum_{k=1}^{N} \beta_{k}=1,
$$

Next, let $G(\lambda) \in \mathbb{R}_{2 N-2}[\lambda]$ be an arbitrary polynomial which is not identically zero and which satisfies the inequality

$$
G(\lambda) \geq 0, \quad-\infty<\lambda<\infty .
$$


This polynomial can be represented in the form (see the proof of Lemma 3)

$$
G(\lambda)=[A(\lambda)]^{2}+[B(\lambda)]^{2},
$$

where $A(\lambda), B(\lambda)$ are polynomials of degrees $\leq N-1$ with real coefficients. Then

$$
\langle\Omega, G(\lambda)\rangle=\sum_{k=1}^{N} \beta_{k} G\left(\lambda_{k}\right)=\sum_{k=1}^{N} \beta_{k}\left[A\left(\lambda_{k}\right)\right]^{2}+\sum_{k=1}^{N} \beta_{k}\left[B\left(\lambda_{k}\right)\right]^{2} \geq 0 .
$$

We have to show that the equality sign in (9.3) is impossible. If we have the equality in (9.3), then, since all the $\beta_{k}$ are positive, we get

$$
A\left(\lambda_{1}\right)=\cdots=A\left(\lambda_{N}\right)=0 \quad \text { and } \quad B\left(\lambda_{1}\right)=\cdots=B\left(\lambda_{N}\right)=0 .
$$

Hence $A(\lambda) \equiv 0$ and $B(\lambda) \equiv 0$ because $\lambda_{1}, \ldots, \lambda_{N}$ are distinct and $\operatorname{deg} A(\lambda) \leq N-1, \operatorname{deg} B(\lambda) \leq$ $N-1$. Therefore we get $G(\lambda) \equiv 0$ which is a contradiction. Finally, if we take

$$
T(\lambda)=\left(\lambda-\lambda_{1}\right) \cdots\left(\lambda-\lambda_{N}\right),
$$

then the condition $(\mathrm{iii})$ of Theorem 7 is also satisfied: for any polynomial $G(\lambda)$,

$$
\langle\Omega, G(\lambda) T(\lambda)\rangle=\sum_{k=1}^{N} \beta_{k} G\left(\lambda_{k}\right) T\left(\lambda_{k}\right)=0
$$

Thus, the functional $\Omega$ defined by the formula (9.2) satisfies all the conditions of Theorem 7 . Therefore there exists a real Jacobi matrix $J$ of the form (1.1), (1.3) for which $\Omega$ is the generalized spectral function. Further, from the proof of sufficiency of the conditions of Theorem 6 it follows that the collection $\left\{\lambda_{k}, \beta_{k}(k=1, \ldots, N)\right\}$ is the spectral data for the recovered matrix $J$.

Note that under the conditions of Theorem 10 the entries $a_{n}$ and $b_{n}$ of the matrix $J$ for which the collection (9.1) is spectral data, are recovered by formulas (3.35), (3.36).

\section{Acknowledgements}

This work was supported by Grant 106 T549 from the Scientific and Technological Research Council of Turkey (TUBITAK).

\section{References}

[1] Boley D., Golub G.H., A survey of matrix inverse eigenvalue problems, Inverse Problems 3 (1987), 595-622.

[2] Ikramov Kh.D., Chugunov V.N., Inverse matrix eigenvalue problems, J. Math. Sciences 98 (2000), 51-136.

[3] Chu M.T., Golub G.H., Inverse eigenvalue problems: theory, algorithms, and applications, Oxford University Press, New York, 2005.

[4] Marchenko V.A., Expansion in eigenfunctions of non-selfadjoint singular second order differential operators, Mat. Sb. 52 (1960), 739-788 (in Russian).

[5] Rofe-Beketov F.S., Expansion in eigenfunctions of infinite systems of differential equations in the nonselfadjoint and selfadjoint cases, Mat. Sb. 51 (1960), 293-342 (in Russian).

[6] Guseinov G.Sh., Determination of an infinite non-selfadjoint Jacobi matrix from its generalized spectral function, Mat. Zametki 23 (1978), 237-248 (English transl.: Math. Notes 23 (1978), 130-136).

[7] Guseinov G.Sh., The inverse problem from the generalized spectral matrix for a second order non-selfadjoint difference equation on the axis, Izv. Akad. Nauk Azerb. SSR Ser. Fiz.-Tekhn. Mat. Nauk (1978), no. 5, 16-22 (in Russian). 
[8] Kishakevich Yu.L., Spectral function of Marchenko type for a difference operator of an even order, Mat. Zametki 11 (1972), 437-446 (English transl.: Math. Notes 11 (1972), 266-271).

[9] Kishakevich Yu.L., On an inverse problem for non-selfadjoint difference operators, Mat. Zametki 11 (1972), 661-668 (English transl.: Math. Notes 11 (1972), 402-406).

[10] Bender C.M., Making sense of non-Hermitian Hamiltonians, Rep. Progr. Phys. 70 (2007), 947-1018, hep-th/0703096.

[11] Znojil M., Matching method and exact solvability of discrete PT-symmetric square wells, J. Phys. A: Math. Gen. 39 (2006), 10247-10261, quant-ph/0605209.

[12] Znojil M., Maximal couplings in PT-symmetric chain models with the real spectrum of energies, J. Phys. A: Math. Theor. 40 (2007), 4863-4875, math-ph/0703070.

[13] Znojil M., Tridiagonal $P T$-symmetric $N$ by $N$ Hamiltonians and fine-tuning of their observability domains in the strongly non-Hermitian regime, J. Phys. A: Math. Theor. 40 (2007), 13131-13148, arXiv:0709.1569.

[14] Allakhverdiev B.P., Guseinov G.Sh., On the spectral theory of dissipative difference operators of second order, Mat. Sb. 180 (1989), 101-118 (English transl.: Math. USSR Sbornik 66 (1990), 107-125).

[15] Guseinov G.Sh., Completeness of the eigenvectors of a dissipative second order difference operator, J. Difference Equ. Appl. 8 (2002), 321-331.

[16] van Moerbeke P., Mumford D., The spectrum of difference operators and algebraic curves, Acta Math. 143 (1979), 93-154.

[17] Sansuc J.J., Tkachenko V., Spectral parametrization of non-selfadjoint Hill's operators, J. Differential Equations 125 (1996), 366-384.

[18] Egorova I., Golinskii L., Discrete spectrum for complex perturbations of periodic Jacobi matrices, J. Difference Equ. Appl. 11 (2005), 1185-1203, math.SP/0503627.

[19] Atkinson F.V., Discrete and continuous boundary problems, Academic Press, New York, 1964.

[20] Akhiezer N.I., The classical moment problem and some related questions in analysis, Hafner, New York, 1965.

[21] Berezanskii Yu.M., Expansion in eigenfunctions of selfadjoint operators, Translations of Mathematical Monographs, Vol. 17, American Mathematical Society, Providence, R.I., 1968.

[22] Nikishin E.M., Sorokin V.N., Rational approximations and orthogonality, Translations of Mathematical Monographs, Vol. 92, American Mathematical Society, Providence, R.I., 1991.

[23] Teschl G., Jacobi operators and completely integrable nonlinear lattices, Mathematical Surveys and Monographs, Vol. 72, American Mathematical Society, Providence, R.I., 2000. 